\title{
A COURSE IN EXTERIOR BALLISTICS
}

\section{A Course in Exterior Ballistics. Ordnance Textbook. Washington,} Government Printing Office, 1921. 127 pp.

This is one of the books prepared by the Ordnance Department, and is issued "for the information and guidance of all concerned." It is based upon a course in ballistic methods given in the Ordnance School of Application at the Aberdeen Proving Ground by Captain R. S. Hoar during the winter of 1919-20. Captain Hoar was at that time in charge of the Ballistic Section of the Proof Department at the Proving Ground. $\mathrm{He}$ is the real author of the book.

As this is the first attempt to give, in anything like complete form, an exposition of the new ballistic methods developed during the World War in this country, it will be of unusual interest. The criticisms that appear in this review are all intended to be constructive, and are the result of observations made while using the book as one of several texts in a course given at Aberdeen during the next term of the school referred to above.

In Chapters I-IV the author gives what he considers "the irreducible minimum of higher mathematics" necessary for an understanding of the later chapters. As prerequisites the student is supposed to be grounded thoroughly "in algebra, in trigonometry, and to know enough of calculus to appreciate the meaning of a derivative, a differential, and a definite integral." The irreducible minimum consists of partial differentiation, successive approximations, the effect of differential variations, and finite differences. Following Chapter IV the book takes up in succession the following topics: An introduction to modern ballistic methods; the computation of trajectories; the computation of differential corrections; the construction of range tables. Alternate methods and a brief mention of the more involved mathematical processes are given in supplements at the end of the book. These topics will be reviewed in this order.

Of the first four chapters those on successive approximations and finite differences are written clearly and explain these subjects in sufficient detail for a thorough understanding of these methods in subsequent chapters. But in interpolating to find range, time of flight, etc., Lagrange's interpolation formula would serve the purpose quite as well as the one given, is at least as easy to use, and is more easily understood by the average student. The chapter on partial differentiation could have been omitted as it contains nothing which cannot be found in standard texts on calculus with which the student is supposed to have some familiarity. The chapter on differential variations and the subject of differential corrections as later developed are, it seems to me, decidedly inadequate. In my opinion the author should have shown in detail how Major F. R. Moulton first derived the differential equations that must be satisfied by the differences between the coordinates of a point on the standard and those of a point on the disturbed trajectory, how these were subsequently simplified, and how 
Professor Bliss introduced his further modification. The introduction of the system of adjoint equations should be presented step by step. To feel that students should have a drill on partial differentiation before undertaking to read the subsequent chapters with appreciation and to assume, tacitly at least, that nothing more would have to be said about adjoint systems than is given in this book, is somewhat out of proportion. One could not ask for a better introduction to this phase on the subject than is given by Professor Dunham Jackson in his ordnance pamphlet published in August, 1919.

The differential equations of the motion of a projectile are

$$
X^{\prime \prime}=-E X^{\prime}, \quad Y^{\prime \prime}=-E Y^{\prime}-G,
$$

where $E=G H / C$, and primes indicate the order of the derivative with respect to $t$. Of these $G$ is a tabulated function of the resistance divided by the velocity, $H$ is a function of the altitude, and $C$ is the ballistic coefficient. These differential equations as well as those used in calculating differential corrections are integrated by means of numerical integration. The introduction of this process as applied to ballistics in this country is due to Major Moulton. The method may be used in this case to obtain any degree of accuracy provided that the physical data are sufficiently accurate. But actually a trajectory so computed is no more accurate than the tabulated $G$-function, and its accuracy is further limited by the fact that the behavior of a projectile is only approximately characterized by its ballistic coefficient. The newer methods are such a decided advantage, however, over all earlier ones that their acceptance as the best working basis for practical ordnance engineering is not open to question.

It is unfortunate that the tables used in the computation of trajectories and differential corrections are not available to the average reader. They are regarded as confidential by the U. S. Army. To the reader who does not have access to them, these parts of the book will not be more interesting than the solution of problems in spherical trigonometry would be without the proper tables.

One correction that is made unnecessary by the new methods is that for the curvature of the earth. This is accomplished by the introduction of a system of curvilinear coordinates; that is, the distances $x$ are measured along the curved surface of the earth, and the distances $y$ are measured along the extensions of the radii of the earth. In calling attention to the very great superiority of this method of treating the problem it is unfortunate that the author makes a grave error at the bottom of page 67 in stating that the error due to disregarding the curvature of the earth (that is, by regarding the earth as flat) is the enormous quantity $.078 R^{2} \operatorname{ctn} \omega$, where $R$ is the radius of the earth and $\omega$ is the angle of fall. The correct expression is $.000000078 X^{2} \operatorname{ctn} \omega$, where $X$ is the range in meters and $\omega$ is the angle of fall.

This error shows that it might have been of considerable advantage to work out a numerical illustration of each correction formula. Such an error would not have occurred if that had been done. Also practical persons like to know how much error is to be expected from a given source. 
As another instance of the same thing in Chapter XIII a very interesting treatment of the influence of the rotation of the earth upon a projectile leaves the reader in doubt as to whether he should expect a variation of $50 \mathrm{~m}$. or of $150 \mathrm{~m}$. in a given range.

The most recent investigations in ballistics seem to indicate that the effect of yaw has been underestimated. The author mentions the fact that this has usually been allowed for in the ballistic coefficient and that we have only very inaccurate information on the subject at present. Not only is this true, but very slight inaccuracies in the manufacture of two projectiles might be sufficient to cause yaws in different directions of such magnitude that more variation would be produced from this cause than by disregarding entirely corrections which have been laboriously made.

The methods developed in this book, with some variations and additions, have been used by Dr. A. A. Bennett in the construction of new ballistic tables. It was my privilege to use these in the course at Aberdeen. They are easy to understand, easy to use, and from every standpoint are all that could be desired at the present time by the practical ballistician.

Although it is generally conceded that real and lasting contributions to ballistics are made rarely, the use of numerical integration will always stand out as a definite contribution of this period of interest in the subject. Also a very decided advance has been made in the way differential equations have been used to attack problems in ballistics. The book under review is the pioneer in presenting these methods "for the information and guidance of all concerned," and all subsequent books will follow it to a great extent. It is to be hoped that a revision of the book will soon be made, if for no other reason, because of the large number of typographical errors. In spite of the very greatest industry and patience on the part of the author a number of these are still to be found, and the text is manifestly unfair to the author in its present form.

It is not an easy matter to carry through successfully the publication of such a book, especially if one is an army officer and subject to the commands of a superior who may or may not appreciate the character and importance of the work. The Army is fortunate, I think, in having had a man of the author's training, experience, enthusiasm, and ability to get things done, to undertake and carry through such an important contribution to the science of ballistics. Full credit is given to all of the mathematicians whose work forms a part of the text, and it would be unappreciative indeed if full credit were not given to the author for any good result that the publication of the book has had or will have in the future. No course in ballistics would be complete without its use as a text, but it should be used to supplement the old ballistics of Ingalls rather than to replace it. The book cannot be read with facility by the average graduate of West Point because he is unfamiliar with much of the mathematics. It might have the very excellent result of raising the standard of mathematics as taught at West Point.

J. E. Rowe 